\section{Contribuição da ultrassonografia abdominal para o diagnóstico da doença celíaca em crianças e adolescentes}

\section{The contribution of abdominal ultrasound to diagnosis of celiac disease in children and adolescents}

Eduardo Just da Costa e Silva 1

Giselia Alves Pontes da Silva 2

\section{Abstract}

Objectives:to compared clinical characteristics and ultrasound parameters in a group of children and adolescents recently diagnosed with celiac disease with a control group.

Methods: a clinical study was carried out with a sample of 20 patients recently diagnosed with celiac disease and 35 patients without the disease and with no symptoms of it. Abdominal ultrasound examinations were carried out with all patients. The cases underwent ultrasound examination during the diagnostic investigation phase, when they were still consuming gluten. Ultrasound and Doppler parameters related to the characteristics of intestinal folds, lymphonodular megaly and alterations in splanchnic blood flow were measured. The differences in proportions between the two groups were evaluated using the chi-squared and Fisher's exact test and the difference between means using Student's t test.

Results: the principal clinical manifestations related to celiac disease were digestive ones, such as diarrhea (12/20), abdominal pain (14/20) and swelling of the abdomen (15/20). Patients with celiac disease presented with a larger transverse diameter of the folds of the small intestine ( $p=0.001)$, greater intestinal peristalsis $(p=0.002)$, increased liquid content in the intestines ( $p=0.02)$, and thickening of the intestine wall $(p<0.001)$.

Conclusions: alterations suggestive of poor digestion/absorption (increased diameter of the transverse folds of the small intestine, greater intestinal peristalsis, increased liquid content) and thickening of the intestine wall in patients complaining of digestive problems should alert clinicians to investigate the possibility of celiac disease.

Key words Celiac disease, Ultrasonography, Child, Adolescent
1,2 Programa de Pós-Graduação em Saúde da Criança e do Adolescente. Universidade Federal de Pernambuco. Av. Prof Moraes Rego, s.n. Prédio das Pós-Graduações do CCS. 10 andar. Cidade Universitária. Recife, PE, Brasil. CEP: 50.670-420.

E-mail: eduardojust@ig.com.br

\section{Resumo}

Objetivos: comparar as características clinicas e parâmetros ultrassonográficos em um grupo de crianças e adolescentes, recentemente diagnosticados como portadores de doença celíaca, com um grupo controle.

Métodos: estudo clínico conduzido com amostra de 20 pacientes recentemente diagnosticados com doença celíaca e 35 pacientes assintomáticos sem doença celíaca. Ultrassonografias abdominais foram realizadas em todos os pacientes. Os casos foram submetidos às ultrassonografias durante a fase de investigação diagnóstica, ainda consumindo glúten. Foram avaliados parâmetros ultrassonográficos e ao Doppler, relacionados às características das alças intestinais, linfonodomegalias e alterações no fluxo sanguíneo esplâncnico. As diferenças de proporções entre os grupos foram avaliadas pelos testes do quiquadrado e exato de Fisher e a diferença de médias pelo teste t de Student.

Resultados: as principais manifestações clínicas relacionadas à doença celíaca foram as digestivas, como diarréia (12/20), dor abdominal (14/20) e distensão abdominal (15/20). Pacientes com doença celíaca apresentaram maior diâmetro transverso das alças do delgado $(p=0,001)$, maior peristaltismo intestinal $(p=0,002)$, aumento do conteúdo líquido intestinal $(p=0,02)$, e maior espessura parietal $(p<0,001)$.

Conclusões: alterações sugestivas de má digestão/absorção (aumento do diâmetro transverso das alças do intestino delgado, maior peristaltismo intestinal, aumento do conteúdo líquido) e o espessamento da parede intestinal em pacientes com queixas digestivas devem alertar o clínico para investigar a doença celíaca.

Palavras-chave Doença celíaca, Ultrassonografia, Criança, Adolescente 


\section{Introdução}

Mudanças no perfil clínico da doença celíaca (DC) têm sido observadas nos últimos 20 anos, registrando-se o aumento do número de casos assintomáticos ou com sintomas não relacionados ao tubo digestório, as quais têm contribuído para o diagnóstico tardio da doença e, consequentemente, o aumento do risco de complicações. 1,2

Os métodos utilizados na investigação diagnóstica da DC são a pesquisa de marcadores imunológicos/sorológicos e a biópsia endoscópica da mucosa duodenal e jejunal,3,4 estando a ultrassonografia indicada apenas no esclarecimento diagnóstico de casos suspeitos que apresentam sintomas abdominais.

Várias imagens ultrassonográficas têm sido relacionadas à $\mathrm{DC}$, porém a maioria tem sido observada em estudos conduzidos em pacientes adultos. $5-13$ Tais imagens tem sido agrupadas em quatro diferentes categorias: as indicativas de problemas digestivos e/ou de absorção, imagens relacionadas a alterações da mucosa intestinal, as compatíveis com processo inflamatório e as imagens compatíveis com as complicações da doença.3,14 Dificuldades relacionadas à padronização na avaliação destes parâmetros e o pequeno número de estudos em crianças e adolescentes têm limitado sua aplicação nesta população.

Este estudo teve por objetivo comparar alguns parâmetros da ultrassonografia abdominal em um grupo de crianças e adolescentes recentemente diagnosticados como portadores de DC, em relação a um grupo de crianças e adolescentes sem a doença e sem sintomas digestivos.

\section{Métodos}

Foi realizado um estudo descritivo de corte transversal comparativo, incluindo 20 portadores de DC e 35 indivíduos assintomáticos, com idade entre um e 18 anos. Os participantes foram recrutados em dois hospitais-escola na cidade do Recife (Instituto de Medicina Integral Prof. Fernando Figueira/IMIP e Hospital das Clínicas da Universidade Federal de Pernambuco/HC-UFPE), no período de setembro de 2009 e julho de 2010.

O tamanho amostral foi definido por conveniência, sendo incluídas todas as crianças atendidas nos serviços para investigação da DC e que ao término do processo investigativo tiveram achados histológicos compatíveis com o diagnóstico da doença.

Os controles foram recrutados em enfermarias de cirurgia pediátrica, internados para cirurgias não relacionadas ao abdome, incluindo ortopedia, cirurgia de cabeça e pescoço e plástica. As ultrassonografias foram realizadas no período pré-operatório. Nenhum exame específico para detecção de DC foi realizado neste grupo.

A coleta dos dados relativos aos portadores de DC foi realizada durante o processo de investigação e todos referiram estar consumindo glúten. Todos os casos foram confirmados posteriormente por achados característicos nas biópsias endoscópicas e melhora após restrição dietética.

Foram excluídos do estudo os indivíduos com história de infecções no último mês, cirurgia recente, passado de neoplasia e atopia. No grupo controle também foram excluídos aqueles com queixas abdominais. Os sinais e sintomas clínicos dos portadores de DC foram obtidos dos prontuários médicos.

Os indivíduos foram examinados por um único ultrassonografista pediátrico, utilizando-se um aparelho modelo HD7 (CA, Bothell), com transdutores linear L12-3 e convexo C5-2. O examinador estava ciente de todos os dados clínicos de cada criança. A avaliação inicial foi realizada pelo modo B (ultrassonografia sem auxílio do Doppler), seguida pelo Doppler (ultrassonografia com avaliação do fluxo vascular).

Os linfonodos foram avaliados quanto ao tamanho (dois eixos), presença do eco central e índice de resistência (IR) dos vasos do hilo, além da ocorrência de aglomerados (grupos de três ou mais linfonodos próximos). O diâmetro e espessura parietal das alças do intestino delgado, a velocidade média e IR da veia porta, a velocidade de pico sistólico e IR da artéria mesentérica superior foram igualmente analisados. O peristaltismo intestinal foi considerado aumentado nos casos em que foram visualizadas ondas peristálticas constantes e simultâneas nos quatro quadrantes do abdome, quando examinadas consecutivamente por dez segundos cada. A presença de alças de intestino delgado preenchidas por líquido nos quatro quadrantes foi interpretada como aumento do conteúdo líquido intestinal e o achado de líquido livre abdominal e de invaginações do intestino delgado foram registradas.

Realizou-se a análise da distribuição de frequência das principais características dos casos e grupo controle. Para as variáveis numéricas, foram obtidas as medidas de tendência central (média ou mediana, de acordo com a distribuição dos resultados da variável, após verificação da normalidade), com suas medidas de dispersão. As diferenças de proporções entre os grupos foram testadas pelo teste 
do qui-quadrado e exato de Fisher e a diferença de médias pelo teste $t$ de Student, a um nível de significância de $5 \%$. As análises estatísticas foram feitas com pacote estatístico EPI-INFO para Windows (versão 3.5.1). Este estudo foi aprovado pelo Comitê de Ética em Pesquisa do Instituto de Medicina Integral Professor Fernando Figueira $\left(\mathrm{N}^{\mathrm{o}}\right.$ 1302-08). Os responsáveis por todos os pacientes assinaram termo de consentimento livre e esclarecido.

\section{Resultados}

A Tabela 1 apresenta as características clínicas dos portadores de DC. A mediana de idade foi de 140 meses $(\mathrm{Q} 1-\mathrm{Q} 3=60,5-187,5)$ nos casos e de 76 meses
(Q1-Q3= 55-112) no grupo de comparação. A maioria dos portadores de DC apresentava as manifestações clássicas da doença: diarréia, dor e distensão abdominal. Dentre os sintomas extraintestinais a perda de peso e fraqueza foram as queixas mais frequentes.

Na Tabela 2 observam-se os parâmetros encontrados com maior frequência nos portadores da DC: maior diâmetro transverso das alças do delgado $(p=0,001)$; maior peristaltismo intestinal $(p=0,002)$; aumento do conteúdo líquido intestinal $(p=0,02) \mathrm{e}$ maior espessura parietal $(p<0,001)$. Em relação à avaliação dos linfonodos abdominais e aos parâmetros relacionados à avaliação do fluxo sanguíneo não foram observadas diferenças significativas do ponto de vista estatístico.

Características clínicas dos portadores de doença celíaca. Instituto de Medicina Integral Prof. Fernando Figueira e Hospital das Clínicas da Universidade Federal de Pernambuco. Recife, 2010. (N=20)

\begin{tabular}{|c|c|c|}
\hline Características & $\mathbf{N}$ & $\%$ \\
\hline \multicolumn{3}{|l|}{ Sexo } \\
\hline Masculino & 4 & 20,0 \\
\hline Feminino & 16 & 80,0 \\
\hline \multicolumn{3}{|l|}{ Sintomas iniciais* } \\
\hline \multicolumn{3}{|l|}{ Intestinais } \\
\hline Diarreia & 12 & 60,0 \\
\hline Dor abdominal & 14 & 70,0 \\
\hline Distensão abdominal & 15 & 75,0 \\
\hline Vômitos & 8 & 40,0 \\
\hline Flatulência & 10 & 50,0 \\
\hline \multicolumn{3}{|l|}{ Extra-intestinais } \\
\hline Perda de peso & 12 & 60,0 \\
\hline Anemia & 7 & 35,0 \\
\hline Dermatite herpetiforme & 4 & 20,0 \\
\hline Aftas orais & 6 & 30,0 \\
\hline Queda de cabelo & 5 & 25,0 \\
\hline Fraqueza & 10 & 50,0 \\
\hline
\end{tabular}

*Um caso pode ter mais um sintoma. 
Resultados ultrassonográficos de portadores de doença celíaca e do grupo controle. Instituto de Medicina Integral Prof. Fernando Figueira e Hospital das Clínicas da Universidade Federal de Pernambuco. Recife, 2010.

\begin{tabular}{|c|c|c|c|}
\hline Características & Doença celíaca & Grupo controle & $p$ \\
\hline Diâmetro transverso das alças do delgado $(\mathrm{cm}) \quad \mathrm{Md}\left(\mathrm{Q}_{25}-\mathrm{Q}_{75}\right)$ & $1,40(1,05-1,80)$ & $0,9(0,70-1,20)$ & $<0,001$ \\
\hline Peristaltismo intestinal aumentado n/N (\%) & $8 / 20(40 \%)$ & $2 / 35(5,7 \%)$ & $0,002^{\dagger}$ \\
\hline Aumento do conteúdo líquido intestinal n/N (\%) & $7 / 20(35 \%)$ & $3 / 35(8,6 \%)$ & $0,02^{\dagger}$ \\
\hline Invaginação do intestino delgado n/N (\%) & $2 / 20(10 \%)$ & $0 / 35(0 \%)$ & 0,15 \\
\hline Espessura parietal- alças do intestino delgado $(\mathrm{mm}) \mathrm{Md}\left(\mathrm{Q}_{25}-\mathrm{Q}_{75}\right)$ & $2(1,5-7,0)$ & $1(1,0-1,1)$ & $<0,001$ \\
\hline Diâmetro maior do maior linfonodo mesentérico $(\mathrm{mm}) \mathrm{Md}\left(\mathrm{Q}_{25}-\mathrm{Q}_{75}\right)$ & $12(11-15)$ & $12(8-14)$ & 0,15 \\
\hline Diâmetro menor do maior linfonodo mesentérico $(\mathrm{mm}) \mathrm{Md}\left(\mathrm{Q}_{25}-\mathrm{Q}_{75}\right)$ & $5,0(4-7)$ & $5,5(3-7)$ & 0,18 \\
\hline Índice de resistência dos vasos do hilo linfonodal‡ $M d\left(Q_{25}-Q_{75}\right)$ & $0,56(0,51-0,65)$ & $0,6(0,57-0,68)$ & 0,22 \\
\hline Presença do eco central ₹ n/N (\%) & $13 / 14(92,9 \%)$ & $29 / 29(100 \%)$ & $0,32+$ \\
\hline Aglomerados linfonodais $¥ \mathrm{n} / \mathrm{N}(\%)$ & $11 / 14(78,6 \%)$ & $26 / 29(89,7 \%)$ & $0,29+$ \\
\hline Velocidade média da veia porta $(\mathrm{cm} / \mathrm{s}) \mathrm{Md}\left(\mathrm{Q}_{25}-\mathrm{Q}_{75}\right)$ & $18,8(16-24)$ & $20(18-25)$ & 0,34 \\
\hline Diâmetro da veia porta $(\mathrm{mm}) \mathrm{Md}\left(\mathrm{Q}_{25}-\mathrm{Q}_{75}\right)$ & $7,2(6,1-8,2)$ & $6,7(5,9-8,0)$ & 0,42 \\
\hline Velocidade sistólica-art. mesentérica superior $(\mathrm{cm} / \mathrm{s}) \mathrm{Md}\left(\mathrm{Q}_{25}-\mathrm{Q}_{75}\right)$ & $164,5(141-299)$ & $193(148-280)$ & 0,62 \\
\hline Índice de resistência da artéria mesentérica superior $M d\left(Q_{25}-Q_{75}\right)$ & $0,84(0,82-0,87)$ & $0,86(0,81-0,89)$ & 0,36 \\
\hline Diâmetro da artéria mesentérica superior $(\mathrm{mm}) \mathrm{Md}\left(\mathrm{Q}_{25}-\mathrm{Q}_{75}\right)$ & $4,6(4,1-5,0)$ & $4,2(3,7-5,1)$ & 0,27 \\
\hline Líquido livre abdominal n/N (\%) & $2 / 20(10 \%)$ & $3 / 35(8,6 \%)$ & $0,60^{+}$ \\
\hline
\end{tabular}

Md $\left(Q_{25}-Q_{75}\right)=$ mediana (Quartis 25-75); † Teste exato de Fisher; ₹ Linfonodos observados em 14 do grupo doença celíaca e em 29 indivíduos do grupo controle; $\mathrm{n}=$ número de casos; $\mathrm{N}=$ total da amostra.

\section{Discussão}

Observou-se maior espessura e diâmetro das alças intestinais, além de maiores conteúdo líquido e peristaltismo intestinal no grupo com DC quando comparados aos indivíduos assintomáticos. Verificase que as diferenças observadas estão relacionadas aos aspectos ultrassonográficos que podem ser explicados pela má absorção/digestão e alterações da mucosa intestinal. Algumas comparações se tornaram impossíveis, entretanto, devido ao número reduzido de indivíduos avaliados. Algumas tendências podem ser verificadas, como diâmetros do maior linfonodo, índice de resistência dos vasos do hilo linfonodal e volume da vesícula biliar. Embora os valores não tenham atingido o nível de significância estatística, é possível que a inclusão de novos indivíduos tornasse as diferenças mais acentuadas.

Estas observações são concordantes com outros estudos ${ }^{6-9,12,13}$ sendo os achados coerentes com a fisiopatologia da doença e refletem a má digestão/absorção, que aumenta o volume do conteúdo luminal e estimula o peristaltismo. A avaliação destes parâmetros pode, entretanto, apresentar algumas dificuldades técnicas. A ultrassonografia abdominal não produz uma imagem panorâmica do abdome, sendo visualizadas diferentes partes de um todo a cada momento. O peristaltismo, portanto, não é visto ao mesmo tempo em todo o abdome, sendo necessário definir parâmetros válidos para caracterizar o seu aumento e tornar os achados mais consistentes.

Os autores que avaliaram o peristaltismo utilizaram definições diferentes. Castiglione et al.7 consideraram peristaltismo aumentado a visualização de mais de três ondas peristálticas por minuto, enquanto Rettembacher et al.12 definiram como alterado a presença de ondas peristálticas persistentes e marcadas em todo o intestino delgado, sendo o seu modelo seguido por Fraquelli et al. ${ }^{9}$ Dois estudos utilizaram o parâmetro, mas não definiram sua avaliação na metodologia.6,13 Dell'Aquilla et al. ${ }^{8}$ compararam o número de ondas peristálticas/tempo entre pacientes e controles. A despeito das diferenças metodológicas, os resultados são semelhantes em mostrar o aumento do peristaltismo em portadores de DC.

Aumento do conteúdo líquido intestinal e dilatação do intestino delgado são parâmetros avaliados de forma separada por diferentes autores $6-9,12,13$ e neste estudo, mas são muito relacionadas. Os achados refletem a expressão gráfica da má absorção/digestão, o que é coerente com o quadro clínico dos pacientes do estudo, que predominaram na forma clássica. 
Uma dificuldade na avaliação destes parâmetros é a exigência do jejum, quando o ideal seria padronizar a ingestão de líquido e alimentos antes da realização do exame, uma vez que sua presença influencia o conteúdo intestinal. A própria prática de jejum antes de ultrassonografias pediátricas tem sido questionada por prejudicar a avaliação desses parâmetros relacionados com a má-absorção/ digestão. ${ }^{15,16}$

A avaliação das pregas do intestino delgado não foi incluída neste estudo, pois seria necessária a ingestão de fluido abundante, com consequentes alterações do peristaltismo e distensão intestinal, o que invalidaria os dois principais parâmetros ora analisados. No entanto, como foi observado aumento da espessura parietal nos portadores de DC, a avaliação das pregas intestinais deve ser feita em estudos subsequentes, pois também refletem alterações mucosas.

As alterações na circulação esplâncnica verificadas por estudos prévios não foram observadas em nossos pacientes, sendo usualmente atribuídas a alterações do fluxo pelo processo inflamatório induzido pela doença. 5,10,17 Destes estudos, o único que incluiu crianças foi o de Ertem et al. 17 Uma possível explicação para os nossos resultados é a diferença de faixa etária dos indivíduos em relação aos demais estudos. Além das alterações do fluxo sanguíneo relacionadas à faixa etária, crianças pequenas se mostram agitadas durante o exame, com consequentes alterações de frequência cardíaca e pressão arterial. Mesmo em relação ao estudo que incluiu crianças, 17 elas foram descritas como examinadas respirando calmamente, o que não ocorreu entre as crianças ora estudadas, coincidindo com a prática diária da ultrassonografia pediátrica.

A associação entre linfonodomegalias abdominais e DC tem sido observada por alguns autores. 6,7,9,12 Rettembacher et al.12 observaram linfonodomegalias em $82 \%$ dos seus pacientes com a doença, mas não fazem referência ao número de controles com este achado, além de não mencionarem se houve diferença estatística no

\section{Referências}

1. Garampazzi A, Rapa A, Mura S, Capelli A, Valori A, Boldorini R, Oderda G. Clinical pattern of celiac diseases is still changing. J Pediatr Gastroenterol Nutr. 2007; 45: 611-

2. Lebenthal E, Shteyer E, Branski D. The changing clinical presentation of celiac disease. In: Fasano A, Troncone R, Branski D, editors. Frontiers in Celiac Disease. Pediatric and Adolescent Medicine. Basel: Karger; 2008. p. 18-22. diâmetro dos linfonodos. Fraquelli et al. ${ }^{9}$ observaram que o achado de linfonodomegalias teve valores preditivos positivo e negativo de $55 \%$ e $95 \%$, respectivamente, para o diagnóstico de DC. Castiglione et al. 7 observaram mais linfonodos mesentéricos em pacientes portadores de DC do que em indivíduos controles, chamando atenção a baixa freqüência de detecção de linfonodos no grupo controle (1/50). Dell'Áquilla et al. ${ }^{8}$ não avaliaram a presença de linfonodos, mas sim de linfonodomegalias, definidas como diâmetro maior do que $0,3 \mathrm{~cm}$ (não especificado se longitudinal ou transverso), com resultados que mostraram diferença estatística entre os grupos, sendo negativos todos os indivíduos do grupo controle.

Nossos resultados não mostraram diferenças entre os parâmetros avaliados associados aos linfonodos. É possível que o processo inflamatório associado à fisiopatologia da DC fique restrito à mucosa intestinal, o que poderia justificar alguns dos achados acima descritos, como espessamento parietal de alças, porém não chegando a atingir os linfonodos. Outra explicação possível é que as alterações de fato ocorram em nível de gânglios, mas sem expressão nos parâmetros atualmente disponíveis na avaliação ultrassonográfica.

As principais limitações do nosso estudo incluem o pequeno tamanho amostral, a possibilidade de inclusão de portadores de doença celíaca entre os controles, ausência de padronização na literatura sobre alguns pontos avaliados, como peristaltismo e o fato de não ser um estudo cego. Novos estudos poderão, todavia, se beneficiar destes resultados, para cálculo de tamanho amostral, permitindo, assim, avaliação dos parâmetros usuais de estudos de acurácia, como sensibilidade, especificidade, valores preditivos e curvas ROC.

Como conclusão, os achados ultrassonográficos de aumento do diâmetro, conteúdo líquido, espessura parietal e peristaltismo das alças do intestino delgado podem ser úteis ao sugerir o diagnóstico de doença celíaca em crianças no processo de investigação diagnóstica de queixas abdominais.

3. Buckley O, Brien JO, Ward E, Doody O, Govender P, Torreggiani WC. The imaging of celiac disease and its complications. Eur J Radiol. 2008; 65: 483-90.

4. Kotz LMS. Doença celíaca. J Bras Gastroenterol. 2006; 6: 23-34.

5. Arienti V, Califano C, Brusco G, Boriani L, Biagi SM, Sottili S, Domanico A, Corazza GR, Gasbarrini G. Doppler ultrasonographic evaluation of splanchnic blood flow in coeliac disease. Gut. 1996; 39: 369-73. 
6. Bartusek D, Valek V, Husty J, Uteseny J. Small bowel ultrasound in patients with celiac disease. Eur J Radiol. 2007 63: 302-6.

7. Castiglione F, Rispo A, Cozzolino A, Camera L, DÁrgenio G, Tortora R, Grassia R, Bucci C, Ciacci C. Bowel sonography in adult celiac disease: diagnostic accuracy and ultrasonographic features. Abdom Imaging. 2007; 32: 73-7.

8. Dell'Aquila P, Pietrini L, Barone M, Cela EM, Valle ND, Amoruso A, Minenna MF, Penna A, De Francesco P, Panella C. Small intestinal contrast ultrasonography-based scoring system: a promising approach for the diagnosis and follow-up of celiac disease. J Clin Gastroenterol. 2005; 39: 591-5.

9. Fraquelli M, Colli A, Colucci A, Bardella MT, Pometta R, Pagliarulo M, Conti D. Accuracy of ultrasonography in predicting celiac disease. Arch Inter Med. 2004; 164: 16974.

10. Giovagnorio F, Picarelli A, Di Giovambattista F, Mastracchio A. Evaluation with Doppler sonography of mesenteric blood flow in celiac disease. Arch Inter Med 1998; 171: 629-32.

11. Magalotti D, Volta U, Bonfiglioli A, Ramilli S, Berzigotti A, Zoli M. Splanchnic haemodynamics in patients with coeliac disease: effects of a gluten-free diet. Dig Liver Dis. 2003; $35: 262-8$

Recebido em 30 de setembro de 2013

Versão final apresentada em 26 de novembro de 2013

Aprovado em 20 de dezembro de 2013
12. Rettembacher T, Hollerweger A, Macheuner P, Huber S, Gritzmann N. Adult celiac disease: US signs. Radiology. 1999; 211: 389-94.

13. Riccabona M, Rossipal E. Sonographic findings in celiac disease. J Ped Gastroenterol Nutr. 1993; 17: 198-200.

14. Rubesin SE, Herlinger H, Saul SH, Grumbach K, Laufer I, Levine MS. Adult celiac disease and its complications. Radiographics. 1989: 9: 1045-66.

15. Rabelo LAAA, Florêncio IR, Pirauá IM, Albuquerque SC, Ribeiro Neto JV, Silva EJC. Do children need fasting before abdominal ultrasonography? Radiol Bras. 2009; 42: 34952.

16. Rocha SMS. Fasting brought into question as a factor influencing the quality of abdominal ultrasonography in children with celiac disease. Radiol Bras. 2009; 42: VIIVIII

17. Ertem D, Tuney D, Baloglu H, Pehlivanoglu E. Superior mesenteric artery blood flow in children with celiac disease. J Ped Gastroenterol Nutr. 1998; 26: 140-5. 